\title{
Inkjet Printed RF Sensor Array For Lung Disease Detection
}

\author{
Muhammad Tayyab ${ }^{1}$, Mohammad S. Sharawi ${ }^{2}$, Atif Shamim ${ }^{3}$, \\ ${ }^{1,2}$ Electrical Engineering Department, King Fahd University for Petroleum and Minerals (KFUPM), Dhahran, 31261 Saudi Arabia, \\ Email: $\{g 201512510$, msharawi $@$ @fupm.edu.sa \\ ${ }^{3}$ Electrical Engineering Department, King Abdullah University of Science and Technology (KAUST), Saudi Arabia \\ Email: atif.shamim@kaust.edu.sa
}

\begin{abstract}
We propose an RF sensor array for lung disease detection. The size of the sensor array is $4 \mathrm{~cm} * 89.4 \mathrm{~cm}$. The sensor consists of 38 electrodes and 37 ports. The first two electrodes are optimized to operate at $60 \mathrm{MHz}$ and act as a signal exciter. The remaining 36 electrodes receive the radiated field. The transmission coefficients $S_{i 1}$ at each passive port are calculated using HFSS software. An equation was designed for dielectric constant estimation using the Least Squares (LS) method. The sensor is fabricated using Inkjet printing. The experiments of the fabricated sensor were conducted on an average adult human chest elliptical model.
\end{abstract}

Index Terms-RF Sensor, LS method, dielectric constant, Inkjet printing, SOC ink .

\section{INTRODUCTION}

The methods of non-invasive imaging of the human body include X-ray, magnetic resonance imaging (MRI) and computed tomography (CT). All these methods are expensive, difficult to carry due to heavy machinery equipments, hospital visit is required and includes many side effects if frequently used. The real time imaging of ventilation and the detection of the collapsed lung is performed using electrical impedance tomography (EIT) [1]. EIT technique is expensive, have large size machinery with high complexity. This technique is limited to bed side applications due to complex circuitry for the generation of the variable current patterns.

Fluid detection measurement and analysis is performed using ultrasound impedance tomography in [2]. The complexity and cost is high. This technique is portable but not wearable. Microwave imaging is used for breast cancer detection and treatment response monitoring in [3]. The technique has small depth of penetration due to high frequency of operation. Because of its complex circuitry, portability and wearability is not possible.

An RF sensor was proposed for pulmonary edema detection in [4]. The size of the sensor was $10 \mathrm{~cm} * 16 \mathrm{~cm}$ with an operating frequency of $40 \mathrm{MHz}$. Different stages of pulmonary edema was detected by estimating the average dielectric constant inside the chest. The sensor consisted of 17 copper electrodes and 16 ports. The cost of the sensor is low with an dielectric constant estimation error of $11 \%$. The sensor have a planner geometry which can monitor the permittivity of limited area.

A flexible RF sensor was proposed in [5] for monitoring deep tissue electrical properties. The operating frequency of the sensor was $40 \mathrm{MHz}$ with a size of $5 \mathrm{~cm} * 83 \mathrm{~cm}$. The sensor was wrapped around a circular cylindrical model of the chest but in reality the human chest model is elliptical. The complexity of the designed RF sensor is high due to subgrouping of the sensor which makes the post processing of the data difficult. The maximum error for dielectric constant estimation was $13.5 \%$.

We propose an RF sensor with low cost and low complexity. The proposed sensor have many features. It is fabricated on a flexible polyimide substrate using an ink of silver organo complex (SOC) with Inkjet printing. The sensor was wrapped around an average adult elliptical human chest model which is the practical case. The size of the sensor is small in width occupying $4 \times 89.4 \mathrm{~cm}^{2}$. The operating frequency of the sensor is optimized to $60 \mathrm{MHz}$ for reducing the human health effects. The depth of penetration is $11.68 \mathrm{~cm}$. The achieved dynamic range between low and high dielectric constant values is 20 $\mathrm{dB}$.

\section{RF SENSOR DESIGN AND FABRICATION}

The proposed RF sensor consist of 38 electrodes and 37 ports. The first two electrodes are optimized to operate at $60 \mathrm{MHz}$ and act like a signal exciter. The first port is the active one and remaining 36 ports are passive which receive the radiated fringing fields. The transmission coefficients $S_{i 1}$ at each passive port are measured. The sensor was fabricated on a thin polyimide sheet of thickness $0.005 \mathrm{~cm}$. The planner sensor geometry is shown in Figure 1. The size of the sensor array is $4 \mathrm{~cm} * 89.4 \mathrm{~cm}$. The elliptical shape of an average human adult chest model is created in CST. The outer chest layer including skin, fat, muscles and bones with average thickness were modeled. The upper torso of human body has a length of $18.29 \mathrm{~cm}$ [6]. The perimeter of an average human chest is $89.4 \mathrm{~cm}$ (which is also the substrate length of the RF sensor) having an elliptical shape with major radius $16.6 \mathrm{~cm}$ and minor 
radius $11.64 \mathrm{~cm} \mathrm{[7].} \mathrm{The} \mathrm{wrap} \mathrm{around} \mathrm{design} \mathrm{of} \mathrm{the} \mathrm{proposed}$ sensor is shown in Figure 2.

A Plexiglas sheet of $1 \mathrm{~cm}$ in thickness is used as a base of the human chest upper torso model. Thin FR-4 sheets $(0.78$ $\mathrm{mm}$ ) were fixed in Plexiglas base for outer and inner layer modeling. The used electrical properties in the proposed sensor design using HFSS are given in Table I. The $S_{i 1}$ curves are collected by changing the $\epsilon_{r}$ inside the inner layer of the chest. Figure 3 shows a comparison of $S_{i 1}$ for different $\epsilon_{r}(1,30$, $60,90,120)$ values with the maximum dynamic range of 20 $\mathrm{dB}$ at port 17 .

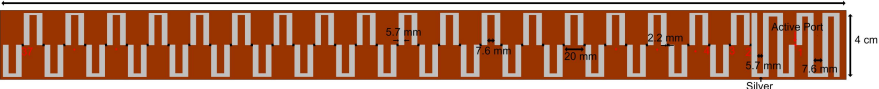

Fig. 1: Proposed design of planner RF sensor

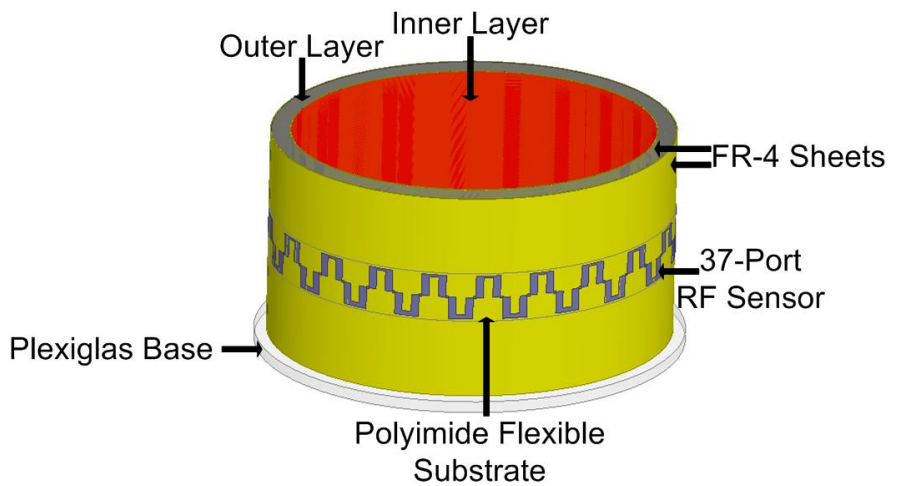

Fig. 2: Wrap around design of the proposed RF sensor on an average adult chest model

TABLE I: Electrical properties of different meterial used for the design of the RF sensor

\begin{tabular}{|c|c|c|c|c|c|}
\hline Material & $\begin{array}{c}\text { Thickness } \\
(\mathrm{cm})\end{array}$ & Permittivity & $\begin{array}{c}\text { Loss } \\
\text { Tangent }\end{array}$ & Conductivity & Reference \\
\hline $\begin{array}{c}\text { Polyimide } \\
\text { sheet }\end{array}$ & 0.005 & 3.4 & 0.01 & - & {$[8]$} \\
\hline FR-4 sheet & 0.07874 & 4.8 & 0.017 & - & {$[9]$} \\
\hline Plexiglas Base & 1 & 2.5 & 0.08 & - & {$[10]$} \\
\hline Outer Layer & 1.5 & 40.6 & 2.33 & 0.38 & {$[4]$} \\
\hline Inner Layer & 31.62 & $1-120$ & $2-3$ & $0.3-0.5$ & {$[4]$} \\
\hline
\end{tabular}

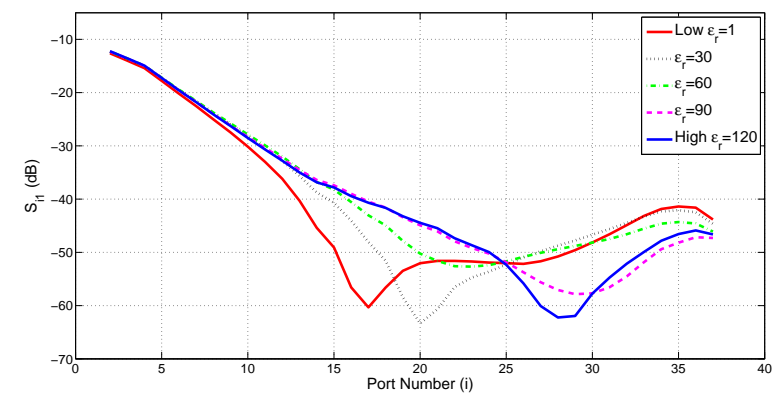

Fig. 3: Comparison of $S_{i 1}$ for different $\epsilon_{r}$ values
The sensor is fabricated using SOC silver ink in an Inkjet printer. The available printer in IMPACT lab, King Abdullah University of Science and Technology, can print A4 size of the sensor. The sensor is divided into five parts. Figure 4 shows the fabrication steps. In first step, the substrate is washed using Isopropyle Alcohol (IPA) as shown in Figure 4(a). The next step is the drying of the substrate using nitrogen gas given in Figure 4(b). The UV light curing of the sensor is optimized to two mints and 30 seconds as shown in Figure 4(c). Increasing the UV time will increase the ink spreading on the substrate. The drop spacing of the SOC silver ink was optimized to 30 micron. After each layer printing, IR bulb heating for five mints was used to cure the silver ink on substrate as shown in Figure 4(d). Due to large size of the substrate, we cannot provide the uniform heating on the whole design. The fabricated five parts of the sensor were joined using double sided tape. The fabricated sensor prototype is shown in Figure 5(a). The sensor on a T-shirt is shown in Figure 5(b) to see its flexibility. The final fabricated prototype of the sensor with coaxial probes is shown in Figure 5(c).

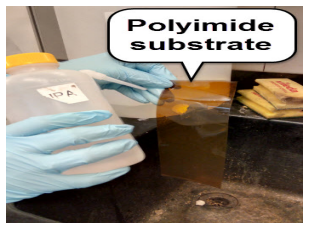

(a)

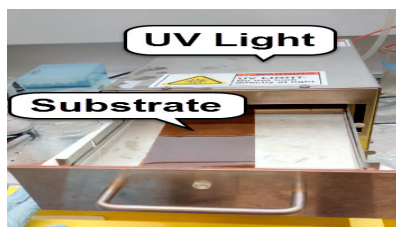

(c)

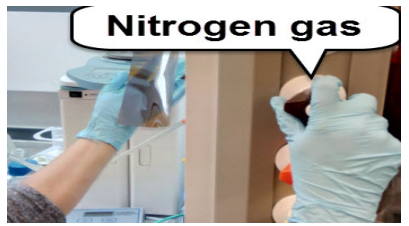

(b)

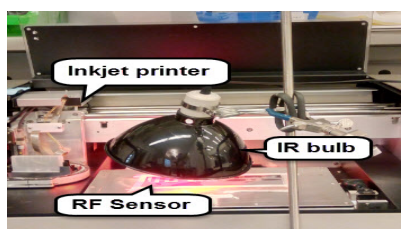

(d)
Fig. 4: RF Sensor printing steps (a) Substarate washing with IPA (b) Drying with nitrogen gas(c) UV light curing (d) IR bulb ink curing

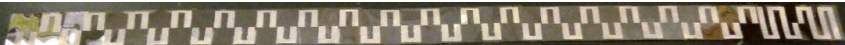

(a)

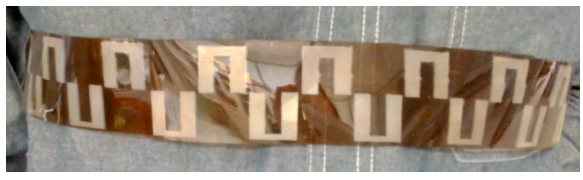

(b)

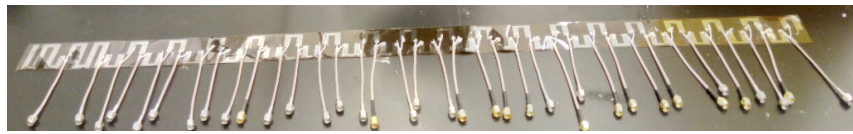

(c)

Fig. 5: Fabricated RF sensor (a) RF sensor without coaxial probes (b) Flexibility of RF sensor (c) RF sensor with coaxial probes. 
The human upper chest torso was designed in the Fablab, KFUPM using computer numerical control (CNC) machine. The plexiglas sheet of $1 \mathrm{~cm}$ in thickness was used for the base design. The 2-D elliptical shape of the chest torso base is programed in VCARVE software. Two $0.5 \mathrm{~cm}$ thickness elliptical groove for outer and inner layer were modeled to fix flexible FR-4 sheet in it. Epoxy glue was used to fix the FR-4 sheets in the grooves. Figure 6 (a) shows the Plexiglas base design on $\mathrm{CNC}$ machine with software commands running on computer. Figure 6(b), (c) and (d) shows the top, front and side view of the designed human upper chest torso, respectively.

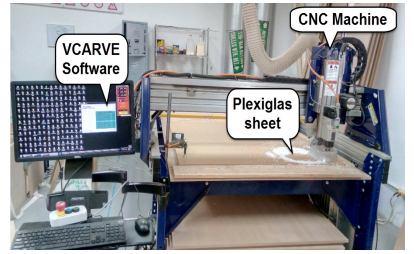

(a)

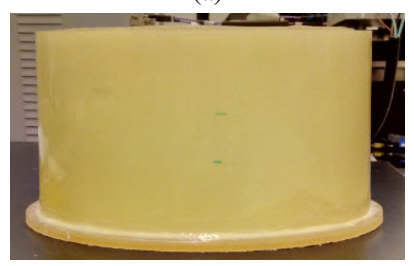

(c)

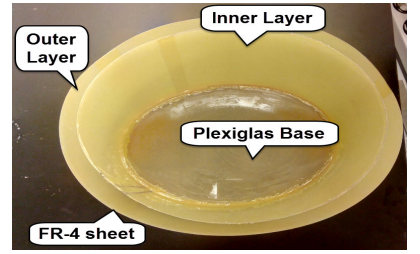

(b)

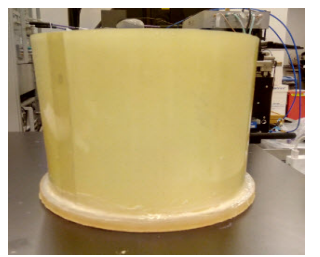

(d)
Fig. 6: Human chest upper torso design (a) Plexiglas base design (b) Top view of the designed upper chest torso (c) front view (d) side view.

\section{Dielectric Constant Estimation}

For dielectric constant estimation, different $S_{i 1}$ values are collected from the simulation model by changing inner layer dielectric constant from 1 to 120 , loss tangent from 2 to 3 and conductivity from 0.3 to $0.5 \mathrm{~s} / \mathrm{m}$. The obtained different 480 $S_{i 1}$ curves are used as a training sequence in the LS method. The retrieved data from HFSS were post processed in a single excel file. MATLAB was used to solve the overdetermined matrix of 480 rows and 36 columns for an operating frequency of $60 \mathrm{MHz}$ as shown in (2). The dielectric constant $\epsilon_{r}$ can be estimated using (1) [5] where $i$ is the port number, $w_{i-1}$ are the weight coefficients (need to determine using LS method) and $S_{i 1}$ are the transmission coefficients. The overdetermined matrix was solved using LS method to obtain the weight coefficients. An equation (3) was obtained for dielectric constant estimation which also includes the weight coefficients.

$$
\epsilon_{r}=\sum_{i=2}^{37} w_{i-1} S_{i 1}
$$

$$
\begin{aligned}
& {\left[\begin{array}{ccccc}
S_{2,1} & S_{3,1} & S_{4,1} & \ldots & S_{37,1} \\
S_{2,1} & S_{3,1} & S_{4,1} & \ldots & S_{37,1} \\
\vdots & \vdots & \vdots & \ddots & \vdots \\
S_{2,1} & S_{3,1} & S_{4,1} & \ldots & S_{37,1}
\end{array}\right] \cdot\left[\begin{array}{c}
w_{1} \\
w_{2} \\
\vdots \\
w_{36}
\end{array}\right]=\left[\begin{array}{c}
\epsilon_{r 1} \\
\epsilon_{r 2} \\
\vdots \\
\epsilon_{r 120}
\end{array}\right] } \\
& \epsilon_{r}= \\
&+7.52 S_{6,1}-4.88 S_{7,1}+0.79 S_{8,1}-4.68 S_{9,1} \\
&+4.74 S_{10,1}-9.83 S_{11,1}+15.11 S_{12,1}-13.11 S_{13,1} \\
&+11.8 S_{14,1}+3.01 S_{15,1}+12 S_{16,1}-17.62 S_{17,1} \\
&+7.45 S_{18,1}-25.04 S_{19,1}+20.31 S_{20,1}+7.53 S_{21,1} \\
&-26.62 S_{22,1}+13.77 S_{23,1}+5.92 S_{24,1}+0.15 S_{25,1} \\
&-2.09 S_{26,1}-0.02 S_{27,1}-3.64 S_{28,1}-10.36 S_{29,1} \\
&+14.64 S_{30,1}-3.78 S_{31,1}+0.01 S_{32,1}-1.32 S_{33,1} \\
&+3.76 S_{34,1}-5.96 S_{35,1}+3.32 S_{36,1}-6.29 S_{37,1}
\end{aligned}
$$

Equation (2) was tested for simulated $S_{i 1}$ curves for normal human lung, edema and emphysema infected lung $\epsilon_{r}$ values (stated in [4]) to see the accuracy of the equation. Table II shows the comparison of exact vs simulated $\epsilon_{r}$ for normal human lung and infected lung. Equation (2) can estimate the $\epsilon_{r}$ of the simulated $S_{i 1}$ with an average error of $0.85 \%$. This error is due to estimation of weight coefficient using LS method.

TABLE II: Comparison of exact vs simulated $\epsilon_{r}$ for normal human lung and with infected lung.

\begin{tabular}{|c|c|c|c|}
\hline Category & $\begin{array}{c}\text { Exact } \\
\epsilon_{r}\end{array}$ & $\begin{array}{c}\text { Simulated } \\
\epsilon_{r}\end{array}$ & $\begin{array}{c}\text { Simulated } \\
\text { Error (\%) }\end{array}$ \\
\hline \hline Normal Lung & 34.2 & 33.74 & 1.35 \\
\hline Emphysema & 19.5 & 19.47 & 0.17 \\
\hline Edema & 51.2 & 51.74 & 1.05 \\
\hline
\end{tabular}

\section{EXPERIMENTAL RESUlTS}

The outer layer electrical properties of the human chest are formulated using a mixture of glycerin, water, salt and IPA. A mixture of $1190 \mathrm{ml}$ IPA, $850 \mathrm{ml}$ glycerin, $340 \mathrm{ml}$ water and $85 \mathrm{~g}$ salt is filled in outer layer of the upper chest torso. The measured $\epsilon_{r}$ using dielectric assessment kit (DAK) was 40.6 for the outer layer of the chest model. The inner layer of the chest model was filled with water. The experimental setup for this case is shown in Figure 7(a). Vector network analyzer (VNA) port $\mathrm{A}$ is connected with the port 1 of the RF sensor (the active port) and VNA port B is switched to other passive ports to collect $S_{i 1}$. Each port reading is taken three times to reduce the measurements inaccuracies and the average measurement is considered for dielectric constant estimation using (2). Figure 7(b) shows the comparison of average measured and simulated $S_{i 1}$. The average measured curve is far away from the simulated one.

To confirm the issue of the fabricated sensor, another measurement was conducted for water and salt mixture in the inner layer of the chest model. The $9000 \mathrm{ml}$ water with $3.15 \mathrm{~g}$ of salt is used for this case in inner layer. The obtained $\epsilon_{r}$ is 108 
which is similar to porcine lung properties [4]. Figure 8(a) shows the measurement setup for water and salt solution in inner layer of the chest and Figure8(b) shows the comparison of average measured and simulated $S_{i 1}$. The average measured $S_{i 1}$ are not matching with the simulation. Table III shows a comparison of exact vs simulated and average measured $\epsilon_{r}$ for water only and water+salt solution test. The average measured error is $33.15 \%$ which is very high.

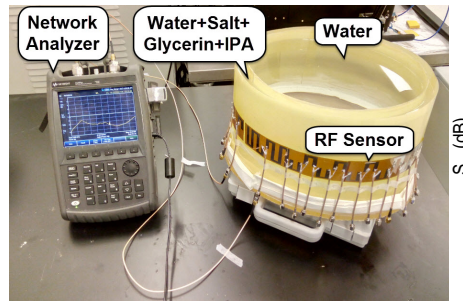

(a)

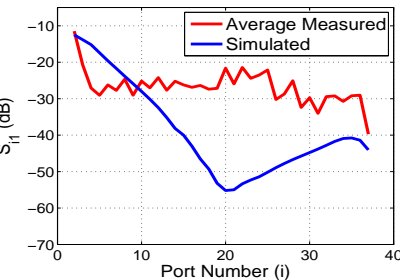

(b)
Fig. 7: Water test (a) Measurement setup (b) comparison of average measured and simulated $S_{i 1}$.

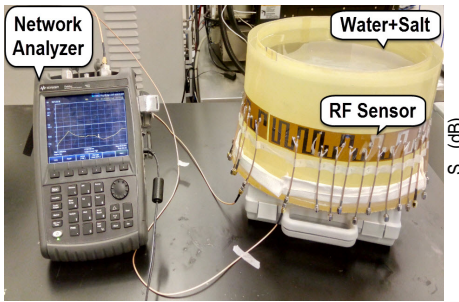

(a)

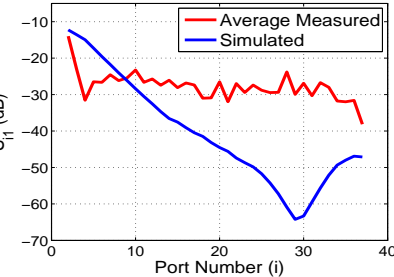

(b)
Fig. 8: Water and salt solution case (a) Measurement setup (b) comparison of average measured and simulated $S_{i 1}$.

TABLE III: Comparison of exact vs simulated and average measured $\epsilon_{r}$ for water only and water+salt solution test.

\begin{tabular}{|c|c|c|c|c|c|}
\hline Category & $\begin{array}{c}\text { Exact } \\
\epsilon_{r}\end{array}$ & $\begin{array}{c}\text { Simulated } \\
\epsilon_{r}\end{array}$ & $\begin{array}{c}\text { Measured } \\
\epsilon_{r}\end{array}$ & $\begin{array}{c}\text { Simulated } \\
\text { Error (\%) }\end{array}$ & $\begin{array}{c}\text { Measured } \\
\text { Error (\%) }\end{array}$ \\
\hline \hline Water+Salt & 108 & 109.31 & 70.12 & 1.21 & 35 \\
\hline Water & 81 & 80.34 & 106.4 & 0.82 & 31.3 \\
\hline
\end{tabular}

The measured $S_{i 1}$ curves are far away from the simulated ones. This is due to non-uniform sliver printing on the polyimide substrate using the Inkjet printer. The second reason is the non uniform IR bulb curing of the silver ink. We are currently trying to re-fabricate and optimize the sensor using advanced nano products (ANP) silver ink. The enlarged view of the first fabricated electrode is shown in Fig. 9(a) which clearly shows the nonuniform and irregular sliver layers on electrodes using SOC silver ink. Figure 9(b) shows the fabricated sensor using ANP ink and the inkjet printer. ANP sliver ink can print uniform ink layers on the polyimide substrate. We are currently fabricating another design of the same sensor using ANP silver ink for results improvement.

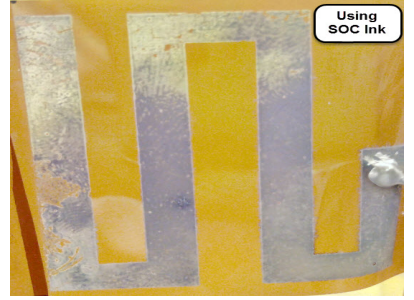

(a)

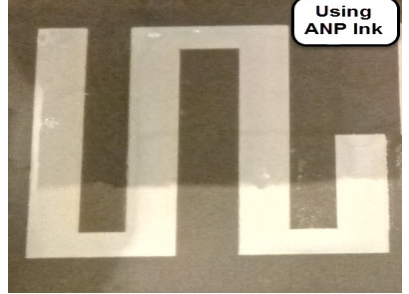

(b)
Fig. 9: Inkjet Printing (a) using SOC silver ink (b) using ANP silver ink.

\section{Conclusions}

A low cost, low complexity and non-invasive sensor is proposed. The sensor size is very small. The sensor was wrapped on an average human adult chest elliptical model for the first time. Simulation results shows errors less than $1.5 \%$. Two experiments were conducted for the fabricated sensor using SOC ink on a polyimide substrate with an average error of $33.15 \%$. The high error percentages are due to nonuniform ink printing on polyimide substrate using SOC silver ink. For the results improvements, we are currently re-fabricating the sensor using ANP silver ink whose printing is much better than the SOC. This has been proven via a single electrode fabrication.

\section{ACKNOWLEDGMENT}

This project was funded by the National Plan for Science, Technology and Innovation (Maarifah) - King Abdulaziz City for Science and Technology - through the Science and Technology Unit at King Fahd University of Petroleum and Minerals (KFUPM) - the Kingdom of Saudi Arabia, under grant number 15-MED-3742-04. And in part by KAUST Award No. OSR 2016-KKI-2899.

\section{REFERENCES}

[1] S. Salman, A Wearable Real-Time and Non-Invasive Thoracic Cavity Monitoring System, PHD Thesis, The Ohio State University, 2015.

[2] S. N. Narouze,Atlas of Ultrasound-Guided Procedures in Interventional Pain Management, Springer, 2011.

[3] P. M. Meaney, P. A. Kaufman, L. S. Muffly, M. Click, S. P. Poplack, W. A. Wells, G. N. Schwartz, R. M. d. F. Alexander, T. D. Tosteson, Z. Li, S. D. Geimer, M. W. Fanning, T. Zhou, N. R. Epstein and K. D. Paulsen, Microwave imaging for neoadjuvant chemotherapy monitoring: initial clinical experience, Breast Cancer Research, (in press).

[4] S. Salman, Z. Wang, E. Colebeck, A. Kiourti, E. Topsakal and J. L. Volakis, Pulmonary Edema Monitoring Sensor With Integrated Body-Area Network for Remote Medical Sensing, IEEE Transaction on antenna and propagation, vol. 62, no. 5, pp. 2787-2794, May 2014.

[5] S. Salman, Lanlin Z. Lee and J. L. Volakis, A Wearable Wrap-Around Sensor for Monitoring Deep Tissue Electric Properties, IEEE sensor journal, vol. 14, no. 8, pp. 2447-2451, August 2014.

[6] E. P. Hanavan, A mathematical model of the human body, Behavioral science laboratory, Air force base, Ohio, Oct 1964.

[7] Available online, https://msis.jsc.nasa.gov/sections/section03.htm

[8] Available online, http://www.goodfellow.com/A/Polyimide-Film.html

[9] Available online, http://www.americanepoxy.com/g10-fr4-datasheet.html

[10] Plexiglas sheet, General information and physical properties of Plexiglas acrylic sheet, Technical data-sheet. 